\title{
REMOVAL OF CONGO RED DYE FROM AQUEOUS SOLUTION BY DATE PALM LEAF BASE
}

\author{
${ }^{1,2}$ Ghadah Alsenani \\ ${ }^{1}$ Department of Chemistry, College of Science, \\ ${ }^{2}$ Deanship of Scientific Research, \\ Princess Nora Bint Abdul Rahman University, Riyadh, KSA
}

Received 2013-01-16; Revised 2014-07-15; Accepted 2014-07-25

\begin{abstract}
Removal of dyes from wastewater is important for industry and environmental protection. The adsorption technique, using biomass is attractive method for environmental and economical reasons. This study studies removal of Congo Red dye (CR) from aqueous solutions by adsorption using date palm Leaf Base (LB). The adsorption of congo red dye $\mathrm{CR}$ onto $\mathrm{LB}$ has been examined in aqueous solution by considering the influence of temperature up to $60^{\circ} \mathrm{C}$ and $\mathrm{pH}$ on the percentage removal of $\mathrm{CR}$. The results showed that the percentage removal of $\mathrm{CR}$ increased with increasing temperature and decreased with increasing $\mathrm{pH}$. The Langmuir and Freundlich models have been applied to describe the equilibrium data and the thermodynamic parameters, Gibbs free energy $\Delta \mathrm{G}^{\circ}$, enthalpy change $\Delta \mathrm{H}^{\circ}$ and entropy $\Delta \mathrm{S}^{\circ}$, have been determined. The negative value of $\Delta \mathrm{H}$ suggests that adsorption of $\mathrm{CR}$ by the date palm leaf base is exothermic. The positive values of $\Delta \mathrm{G}^{\circ}$ at all studied temperatures indicate that $\mathrm{CR}$ adsorption is a unspontaneous process. $\mathrm{LB}$ is a promising adsorbent for the removal of $\mathrm{CR}$ from aqueous solution over a range of concentrations.
\end{abstract}

Keywords: Adsorption, Date Palm Leaf Base, Congo Red, Thermodynamic Parameters

\section{INTRODUCTION}

Dyes are used extensively in textile industries. The wastewater from dyeing and finishing operations in the textile industry is generally high in both color and organic content. Exposure to Cong Red dye (CR) can cause allergic reactions. CR contains aromatic amine groups, which are suspected carcinogens (Mozumder and Islam, 2010). A cost-effective dye removal technique is therefore important for industry and environmental protection. The adsorption technique, using different types of adsorbents, is superior to other techniques because of its efficacy, economy, ability to separate a wide range of chemical compounds and easy operational procedures. Recently, researchers have focused on the use of biomaterials as adsorbents for environmental and economical reasons (Khaled et al., 2009; Dávila-Jiménez et al., 2009; Hameed et al., 2009; Tunc et al., 2009; Hameed et al., 2008; Hameed and
El-Khaiary, 2008). Considering that date palm is one of the most cultivated palms around the world, especially in Arab and Middle Eastern countries, utilizing date palm waste to develop new adsorbents for wastewater treatment by adsorption is quite attractive. Thus, this study studies CR removal from aqueous solutions by adsorption using date palm Leaf Base (LB). Removal of day as a function of temperature and $\mathrm{pH}$ are investigated and the thermodynamic parameters are calculated.

\section{EXPERIMENTAL}

\subsection{Materials}

Date palm Leaf Base (LB) was obtained locally from a farm in the southern region of Riyadh city in Saudi Arabia. It was collected, sorted, cut, ground and sieved to obtain a fine powder. The powder was then kept dry in a closed container until use. No chemical or physical treatments

Corresponding Author: Ghadah Alsenani, Department of Chemistry, College of Science,

Princess Nora Bint Abdul Rahman University, Riyadh, KSA 
were performed prior to the adsorption experiments. The Congo Red (CR) used as an adsorbate was supplied by Techno Pharmchem (Bahadurgarh, India).

\subsection{Methods}

For the adsorption study, distilled water was used to prepare various solutions at the desired concentrations from the stock solution. For each individual test, $0.5 \mathrm{~g}$ of the adsorbent was placed into a screw-capped Erlenmeyer flask containing $25 \mathrm{~mL}$ of different concentrations of CR, from $5 \times 10^{-5}$ to $4 \times 10^{-4} \mathrm{~mol} / \mathrm{L}$. The flasks were shaken for a sufficient period to achieve equilibrium at the unadjusted solution $\mathrm{pH}$. The mixture was filtered and the dye uptake was monitored spectrophotometrically (Jenway model 6800 UV/VIS spectrophotometer) by measuring the absorbance at the $\lambda_{\max }$ of $530 \mathrm{~nm}$. The adsorption at equilibrium, $q_{e}$ $(\mathrm{mol} / \mathrm{g})$, was calculated by:

$q_{e}=\frac{\left(c_{0}-c_{e}\right) v}{m}$

where, $C_{0}$ and $C_{e}(\mathrm{~mol} / \mathrm{L})$ are the liquid-phase dye concentrations initially and at equilibrium, respectively. $V$ is the volume of the solution $(L)$ and $m$ is the mass of the dry adsorbent $(g)$. The equilibrium data were then fitted using the Langmuir and Freundlich isotherm models.

The effect of $\mathrm{pH}$ on the dye adsorption process was investigated by batch equilibrium studies at different $\mathrm{pH}$ values in the range of 3-10.

The effect of temperature was studied by calculating the percentage removal of $4 \times 10^{-4} \mathrm{~mol} \mathrm{~L}^{-1}$ dye solution at temperatures up to $60^{\circ} \mathrm{C}$.

The thermodynamic parameters calculated to describe the adsorption process onto LB include the changes in standard enthalpy $\left(\Delta \mathrm{H}^{\mathrm{o}}\right)$, standard entropy $\left(\Delta \mathrm{S}^{\mathrm{o}}\right)$ and standard free energy $\left(\Delta \mathrm{G}^{\mathrm{o}}\right)$.

\section{RESULTS}

\subsection{Adsorption Isotherms}

The adsorption isotherms indicate how the adsorption molecules are distributed between the liquid phase and the solid phase when the adsorption process reaches equilibrium (Tan et al., 2008). Figure 1 shows the adsorption isotherm of CR onto $\mathrm{LB}$ at $25^{\circ} \mathrm{C}$.
To find a suitable model for design purposes, the isotherm data analysis includes fitting to different isotherm models. This step has been achieved by applying the linear forms of the Langmuir (Equation 1) and Freundlich (Equation 2) adsorption isotherm models (Sivaramakrishna et al., 2014). The applicability of the isotherm equations to describe the adsorption process was judged based on the correlation coefficients $R^{2}$ and the maximum value of adsorption:

$$
\begin{aligned}
& \frac{1}{q_{e}}=\frac{1}{Q_{0}}+\left(\frac{1}{b Q_{0}}\right)\left(\frac{1}{C_{e}}\right) \\
& \log q_{e}=\log K_{f}+\frac{1}{n} \log C_{e}
\end{aligned}
$$

where, $C_{e}$ is the equilibrium concentration, $q_{e}$ is the amount of adsorbate adsorbed per unit mass of adsorbent (mol/g) at equilibriu $\mathrm{m}$ and $Q_{o}$ and $\mathrm{b}$ are Langmuir constants related to the adsorption capacity and rate of adsorption, respectively. $K_{f}$ and $\mathrm{n}$ are Freundlich constants related to the adsorption capacity and adsorption intensity, respectively. The constants for all of the adsorption isotherms are listed in Table $\mathbf{1}$ and the theoretical plots of these isotherms are shown in Fig. 2 and 3.

For the Langmuir model, which suggests monolayer coverage of the dye on the solid surface, plotting $\frac{1}{q_{e}}$ against $\frac{1}{C_{e}}$, as represented in Fig. 2, yields a straight line, confirming its applicability for describing the adsorption of CR over LB. The correlation coefficient $\left(R^{2}\right)$ for the Langmuir model is 0.976 and the value of $\mathrm{Q}_{\mathrm{o}}$ is $0.20 \times 10^{-4} \mathrm{~mol} \mathrm{~g}^{-1}$. Thus, the experimental adsorption data fit this model fairly well.

To confirm the favorability of the process, the dimensionless constant separation factor or equilibrium parameter $\left(R_{L}\right)$ was calculated as follows (Alshabanat et al., 2013):

$$
r=\frac{1}{1+b C_{0}}
$$

where, the value of $b$ was obtained from the Langmuir isotherm. In general, $R_{L}$ indicates the isotherm type: Irreversible $(r=0)$, favorable $(0<r<1)$, linear $(r=1)$ and unfavorable $(r<1)$. The value of $(r)$ in this study is 0.12716 , indicating a favorable isotherm.

The equilibrium data are described according to the Freundlich model in Fig. 3. The correlation coefficient 
$\left(R^{2}\right)$ of the Freundlich model is below 0.90 and the value of the Freundlich constant $K_{f}$, which is related to the adsorption capacity, which is presented in Table $\mathbf{1}$, are much higher than the experimental value.

\subsection{Effect of Temperature}

Figure 4 shows the percentage removal of the dye by the proposed adsorbent. When the temperature is increased from 25 to $60^{\circ} \mathrm{C}$, the removal of $\mathrm{CR}$ by adsorption increases. An increase in temperature is expected to increase adsorption by decreasing the viscosity of the solution and increasing the mobility of the adsorbate.

\subsection{Effect of $\mathbf{p H}$}

The effect of $\mathrm{pH}$ on $\mathrm{CR}$ adsorption onto $\mathrm{LB}$ was studied through tests carried out on solutions of various $\mathrm{pH}$ as shown in Fig. 5, the dye adsorption increased as the $\mathrm{pH}$ of the solution decreased, whereas the further increase of $\mathrm{pH}$ in alkaline solutions hindered the dye adsorption.

\subsection{Thermodynamic Parameters}

The thermodynamic parameters for the adsorption of CR onto LB were calculated using the equilibrium constant for $\mathrm{CR}$ adsorption, $K_{d}$, at different temperatures. The values of this constant were calculated at $25,40,50,60$ and $70^{\circ} \mathrm{C}$ using the following equation (Alshabanat et al., 2013):

$$
K_{d}=\frac{\text { dye concentration in adsorbent at equilibriun }}{\text { dye concentration in solution at equilibrium }}
$$

The thermodynamic parameters, i.e., the changes in standard free energy $\left(\Delta \mathrm{G}^{\circ}\right)$, standard enthalpy $\left(\Delta \mathrm{H}^{\circ}\right)$ and standard entropy $\left(\Delta \mathrm{S}^{\circ}\right)$, were determined using different equations. $\Delta \mathrm{G}^{\circ}$ wascalculated by:

$$
\Delta G=-R T \ln K_{d}
$$

The standard enthalpy $\Delta \mathrm{H}^{\circ}$ and the entropy change were calculated by the Van't Hoff equation:

$$
\ln K_{d}=\frac{\Delta S^{\circ}}{R}-\frac{\Delta H^{\circ}}{R T}
$$

As shown in Fig. 6, was obtained from the slope of the linear plot of $\ln K_{d}$ versus $1 / T$, whereas $\Delta S^{\circ}$ was calculated from the intercept.

Table 2 provides the obtained thermodynamic parameters.

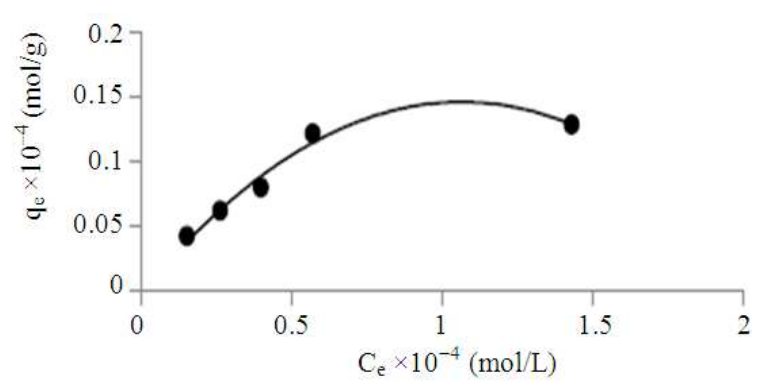

Fig. 1. Adsorption isotherms of $\mathrm{CR}$ on $\mathrm{LB}$

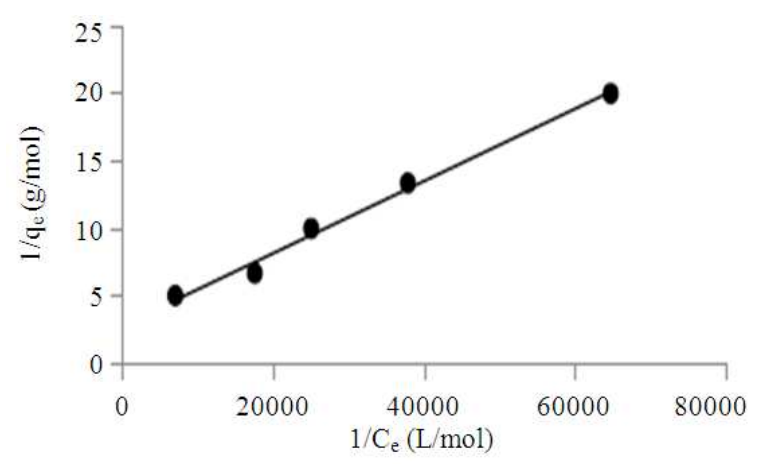

Fig. 2. Langmuir plot for CR adsorption onto LB

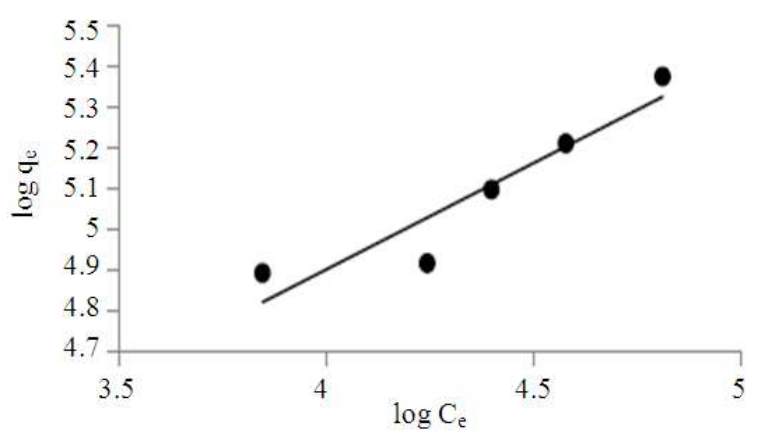

Fig. 3. Freundlich plot for CR adsorption onto LB

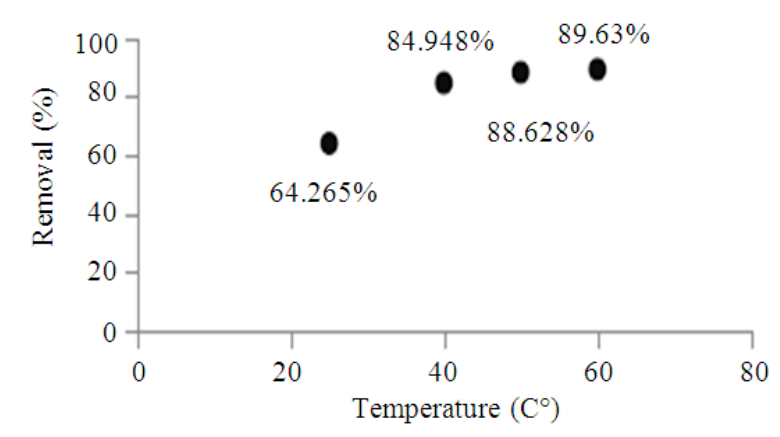

Fig. 4. Effect of temperature on CR adsorption onto LB 


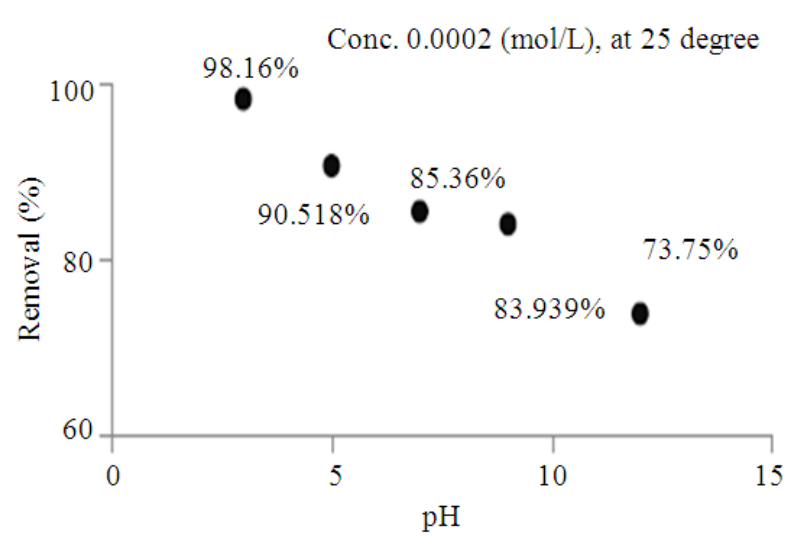

Fig. 5. Effect of $\mathrm{pH}$ on $\mathrm{CR}$ adsorption onto LB

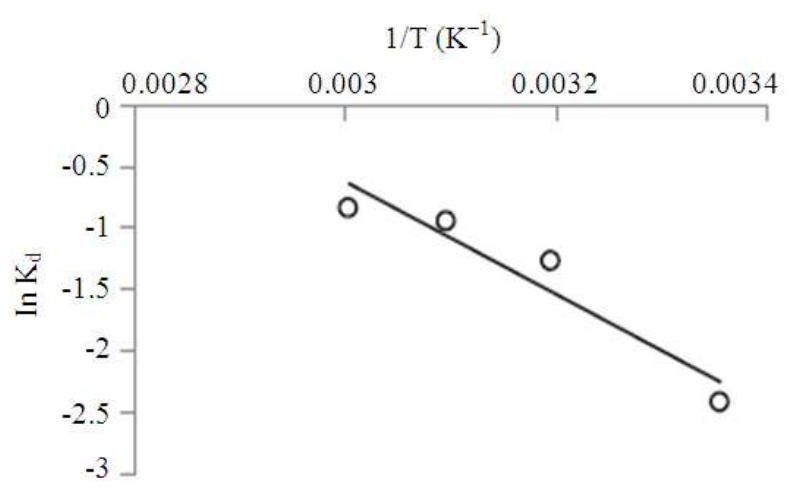

Fig. 6. In Kd versus 1/T according to the Van't Hoffe equation

Table 1. Values of the constants in the different adsorption models

\begin{tabular}{llll}
\hline Langmiur & $\mathrm{R}^{2}$ & $\mathrm{Q}_{\mathrm{e}}(\mathrm{mol} / \mathrm{g})$ & $\mathrm{b}(\mathrm{L} / \mathrm{mol})$ \\
\hline \multirow{3}{*}{ Frindulish } & 0.976 & $0.20 \times 10^{-4}$ & $0.1716 \times 10^{5}$ \\
& $\mathrm{R}^{2}$ & $\mathrm{~K}_{\mathrm{f}}$ & $\mathrm{n}$ \\
& 0.876 & $6.516 \times 10^{2}$ & 1.919 \\
\hline
\end{tabular}

Table 2. Thermodynamic parameters

\begin{tabular}{llll}
\hline $\mathrm{T}(\mathrm{K})$ & $\Delta \mathrm{G}^{\mathrm{o}}(\mathrm{KJ} / \mathrm{mol})$ & $\Delta \mathrm{H}^{\mathrm{o}}(\mathrm{KJ} / \mathrm{mol})$ & $\Delta \mathrm{S}^{\circ}(\mathrm{J} / \mathrm{K} . \mathrm{mol})$ \\
\hline 298 & 5.968 & & \\
313 & 3.290 & & \\
323 & 2.530 & -11272.95 & 108.248 \\
333 & 2.320 & & \\
\hline
\end{tabular}

\section{DISCUSSION}

\subsection{Adsorption Isotherms}

The isotherm is clearly type $L$ according to the Giles classification, which indicates that as more sites in the substrate are filled, it becomes increasingly difficult for a bombarding solute molecule to find a vacant site. This finding implies that there is no strong competition from the solvent (Giles et al., 1960). Regardless of mechanism, the maximum value is $0.13 \times 10^{-4} \mathrm{~mol} \mathrm{~g}^{-1}$.

Regression analysis reveals that the Langmuir model fits the experimental data well with correlation factor higher than 0.97 for adsorbent. This indicates that the adsorption mechanism of CR onto LB can be assumed as monolayer coverage and the adsorption is homogeneous, where the adsorption of each adsorbate molecule onto the surface has equal sorption activation energy.

It is pertinent to mention that the Langmuir model is better fitted than Fraundlich model in this study and indicates the formation of the monolayers of the dye on the adsorbent.

\subsection{Effect of Temperature}

A slight increase in removal of the dye by LB was observed with increasing temperature. This observation revealed that the adsorption process was slightly endothermic. This may be caused by the increased tendency of adsorbate ions mobility with temperature which slightly enhances the removal of CR from the solution by LB (Al-Khatib et al., 2012).

\subsection{Effect of $\mathrm{pH}$}

Acidity is very important in the adsorption process, especially for dye adsorption. The $\mathrm{pH}$ of a medium will control the magnitude of the electrostatic charges imparted by the ionized dye molecules. Both the adsorbent and the adsorbate may contain functional groups that can be protonated or deprotonated to produce different surface charges at different $\mathrm{pH}$, resulting in electrostatic attraction or repulsion between the charged adsorbates and adsorbents (Sivaramakrishna et al., 2014). The lower adsorption of $\mathrm{CR}$ in alkaline media may be due to the presence of excess $\mathrm{OH}$-ions competing with dye anions for the adsorption sites.

\subsection{Thermodynamic Parameters}

The positive free energy values at all temperatures indicate that the process is unspontaneous. The increase in $\Delta \mathrm{G}^{\circ}$ with decreasing temperature suggests greater dye adsorption at high temperatures. The exothermic nature of process was confirmed by the negative value of the enthalpy change $\Delta \mathrm{H}^{\circ}$. Positive values of the entropy change $\Delta S^{\circ}$ indicate the good affinity of the adsorbent towards CR dye. 


\section{CONCLUSION}

Considering the results described above, it can be concluded that CR could be removed onto LB within the used concentration range by adsorption. According to correlation coefficient and experimental and theoretical maximum adsorption capacity, the Langmuir model describes the adsorption process better than the Freundlich model. According to the measurements made in this study, the percentage removal of the dye increases with increasing temperature and decreasing $\mathrm{pH}$.

\section{ACKNOWLEGMENT}

The researchers would like to thank the Deanship of Scientific Research at Princess Nora Bint Abdurrahman University for providing the funding for this study under project number 016-5-31.

\section{REFERENCES}

Al-Khatib, L., F. Fraige, M. Al-Hwaiti and O. AlKhashman, 2012. Adsorption from aqueous solution onto natural and acid activated bentonite. Am. J. Environ. Sci., 8: 510-522. DOI: 10.3844/ajessp. 2012.510.522

Alshabanat, M., G. Alsenani and R. Almufarij, 2013. Removal of crystal violet dye from aqueous solutions onto date palm fiber by adsorption technique. J. Chem., 1: 6-6. DOI: $10.1155 / 2013 / 210239$

Dávila-Jiménez, M.M., M.P. Elizalde-González and V. Hernández-Montoya, 2009. Performance of mango seed adsorbents in the adsorption of anthraquinone and azo acid dyes in single and binary aqueous solutions. Bioresource Technol., 100: 6199-6206. DOI: 10.1016/j.biortech.2009.06.105

Giles, C.H., T.H. Macewan, S.N. Nakhwa and D.J. Smith, 1960. Studies in adsorption. Part XI. A system of classification of solution adsorption isotherms and its use in diagnosis of adsorption mechanisms and in measurement of specific surface areas of solids. J. Chem. Society, 111: 3973-3993. DOI: $10.1039 / J R 9600003973$
Hameed, B.H. and M.I. El-Khaiary, 2008. Removal of basic dye from aqueous medium using a novel agricultural waste material: Pumpkin seed hull. J. Hazardous Mater., 155: 601-609. DOI: 10.1016/j.jhazmat.2007.11.102

Hameed, B.H., D.K. Mahmoud and A.L. Ahmad, 2008. Equilibrium modeling and kinetic studies on the adsorption of basic dye by a low-cost adsorbent: Coconut (Cocosnucifera) bunch waste. J. Hazardous Mater., 158: 65-72. DOI: 10.1016/j.jhazmat.2008.01.034

Hameed, B.H., R.R. Krishni and S.A. Sata, 2009. A novel agricultural waste adsorbent for the removal of cationic dye from aqueous solutions. J. Hazardous Mater., 162: 305-311. DOI: 10.1016/j.jhazmat.2008.05.036

Khaled, A., A. El Nemr, A. El-Sikaily and O. Abdelwahab, 2009. Treatment of artificial textile dye effluent containing direct yellow 12 by orange peel carbon. Desalination, 238: 210-232. DOI: 10.1016/j.desal.2008.02.014

Mozumder, M.S.I. and M.A. Islam, 2010. Development of treatment technology for dye containing industrial waste water. J. Sci. Res., 2: 567-576. DOI: 10.3329/jsr.v2i3.4302

Sivaramakrishna, L., M. Sivasankar Reddy, M. Jagadeesh, W.Y. Wan Zuhairi, M.R. Taha and A. Varada Reddy, 2014. Evaluation of biomass, Indian ujuba Seed (IJS) for removal of congo red. Am. J. Environ. Sci., 10: 374-382. DOI: 10.3844/ajessp.2014.374.382

Tan, I.A.W., A.L. Ahmad and B.H. Hameed, 2008. Adsorption of basic dye on high-surface-area activated carbon prepared from coconut husk: Equilibrium, kinetic and thermodynamic studies. J. Hazardous Mater., 154: 337-346. DOI: 10.1016/j.jhazmat.2007.10.031

Tunc, Ö., H. Tanac1 and Z. Aksu, 2009. Potential use of cotton plant wastes for the removal of remazol black B reactive dye. J. Hazardous Mater., 163: 187-198. DOI: 10.1016/j.jhazmat.2008.06.078 